\title{
Challenging Perceptions About Oncology Product Pricing in Breast and Colorectal Cancer
}

\author{
Anthony Barron ${ }^{1}$ Tim Wilsdon ${ }^{1}$
}

Published online: 10 November 2016

(C) The Author(s) 2016. This article is published with open access at Springerlink.com

\begin{abstract}
Although significant progress has been made in the past decade in the treatment of both common and rare cancers, there has been significant concerns about the cost, and especially the value, of certain new oncology drugs. These concerns touch upon a number of issues regarding the price of these medicines, the value they deliver and the ability of healthcare systems to fund them. This paper looks at these perceptions and the extent to which they apply across different oncology products. Whilst it is acknowledged there is evidence that the launch price of pharmaceutical treatments for some forms of cancer has been rising in recent years, this is not uniformly the case; we find evidence to the contrary for some forms of cancer. This is illustrated by the cases of breast and colorectal cancer. We find cancer medicine prices depend on a number of factors, including pre-existing treatment options within a therapeutic class. Indeed, a number of studies have focused on the cost of treatment per month of overall survival gained as a simple (although partial) metric to judge value for money. Given the importance of oncology products being used in combination, developing similar approaches to capturing the overall cost of treatment will be crucial.
\end{abstract}

Anthony Barron

abarron@crai.com

1 Charles River Associates, Life Sciences, 8 Finsbury Circus, London EC2M 7EA, UK

\section{Key Points}

Looking at cost per month of value gained, evidence for recent drugs indicates that some new oncology drugs are delivering additional value and at a lower cost than pre-existing products.

Given the importance of oncology products being used in combination, developing new approaches to capturing the overall cost of treatment will be crucial.

\section{Current Criticisms on New Oncology Products}

Although significant progress in the treatment of both common and rare cancers has been made in the past decade, there has been significant concern about the cost, and especially the value, of certain new oncology drugs. These concerns touch upon a number of issues regarding the price of these medicines, the value they deliver and the ability of healthcare systems to fund them [1].

Controversies over the value of and 'willingness to pay' for novel therapies have long existed in many high-income countries with publically funded healthcare systems, such as Australia, Canada and the UK. This has led to a number of beliefs and assertions regarding new innovative oncology products:

- Launch prices of new anticancer drugs are significantly and systematically increasing over time, leading to growing concerns about whether the overall cost of care is economically 'unsustainable' and whether this is leading to unacceptable trade-offs for non-oncology patients $[2,3]$. 
- Many oncology advances are not delivering significant benefits to patients or to the healthcare system, leading to the perception that "the cost of the new generation of drugs is getting out of all proportion to the added benefit" [4-8].

- Companies are taking advantage of highly inflexible consumer demand and the pressure on payers to improve access to cancer treatments to ratchet up prices [2] and are applying prices based on "what they can get away with" rather than the intrinsic value of the product [6,8-11].

The high prices of cancer drugs mean the cost of covering new oncology treatments is becoming unaffordable for most health systems, which is crowding out other services from payer budgets [2, 4, 12, 13]. In this paper, we look at the evidence underlying these perceptions, how they apply across different tumour types and whether there are counter-examples and arguments that might help to provide a more balanced understanding of the issue.

\section{What is Happening to Launch Prices?}

Various studies concur that, as a general trend, the launch price of anticancer drugs has been increasing in recent years [4, 10]. A 2013 article published in the Journal of Clinical Oncology found the average monthly price of cancer drugs in the USA had more than doubled from \$US4500 a decade ago to over \$US10,000 [8].

However, there are some examples of recent oncology products where costs for a month of treatment run counter to this trend and are delivering additional value at a lower cost than pre-existing products, reflecting development of in-class competition and the individual role of additional treatment options in each therapy area.

Using the Memorial Sloan Kettering Cancer Center (MSKCC) database (https://www.mskcc.org/research-areas/ programs-centers/health-policy-outcomes/cost-drugs) on cancer drug prices, which compiles monthly treatment costs based on list prices (\$US, year 2014 values), we looked at drug prices for various cancer types (i.e. colorectal, breast cancer). While some drug prices have increased, a careful look at individual therapeutic classes demonstrated that the prices of other drugs are lower than other products in their class.

\subsection{Trends in Absolute Prices Versus Total Treatment Costs}

We find that there are exceptions in pricing trends in colorectal cancer, where more treatment options have recently been introduced for patients with metastatic colorectal cancer (mCRC) along with new ways to combine traditional agents.

As illustrated in Table 1, both panitumumab (a monoclonal antibody targeting tumours that overexpress the epidermal growth factor receptor) and regorafenib (a multikinase inhibitor) are delivering additional value at a cost that is lower than or similar to that of some pre-existing products. Therefore, prices have not been systematically trending upward in this particular class of products.

Similarly, considerable advances have been made in the treatment of certain subtypes of breast cancer. Treatment options for human epidermal growth factor (HER)-2-positive metastatic breast cancers include trastuzumab, pertuzumab, lapatinib, and trastuzumab-emtansine, and many more are in development [14]. As shown in Table 2, the monthly treatment cost of pertuzumab, introduced in 2013, is significantly lower than the monthly cost of trastuzumabemtansine introduced in the same year. In reality, some, although not all, of the new medicines are primarily used in combination; hence, the focus should move from the price of individual medicines to how overall costs are changing.

\subsection{Trends in Value for Money}

A number of recent studies have demonstrated that cost of treatment per month of overall survival (OS) gained can be a useful metric that can be standardised across countries to easily compare the value for money of treatments [15]. Such analysis may be useful to compare different treatments and help shed light on their relative value.

Table 1 Monthly drug prices for colorectal cancer

\begin{tabular}{lllcr}
\hline Generic name & Brand name & $\begin{array}{l}\text { Year of US } \\
\text { FDA approval }\end{array}$ & $\begin{array}{l}\text { Monthly cost } \\
\text { (actual \$US) }\end{array}$ & $\begin{array}{l}\text { Monthly cost } \\
\text { (\$US, year 2014 values) }\end{array}$ \\
\hline Oxaliplatin & Eloxatin & 2002 & 5911 & 7778 \\
Bevacizumab & Avastin & 2004 & 4429 & 5551 \\
Cetuximab & Erbitux & 2004 & 9465 & 11,862 \\
Panitumumab & Vectibix & $\mathbf{2 0 0 6}$ & $\mathbf{7 9 9 1}$ & $\mathbf{9 3 8 4}$ \\
Ziv-aflibercept & Zaltrap & 2012 & 11,063 & 11,407 \\
Regorafenib & Stivarga & $\mathbf{2 0 1 2}$ & $\mathbf{9 6 2 0}$ & $\mathbf{9 9 1 9}$
\end{tabular}

Bold values indicate additional value at lower or similar cost than existing treatments

a Adjusted for inflation 
Table 2 Monthly drug prices for breast cancer

\begin{tabular}{|c|c|c|c|c|}
\hline Generic name & Brand name & $\begin{array}{l}\text { Year of US FDA } \\
\text { approval }\end{array}$ & $\begin{array}{l}\text { Monthly cost } \\
\text { (actual \$US) }\end{array}$ & $\begin{array}{l}\text { Monthly cost } \\
\text { (\$US, year } 2014 \text { values) }^{\text {a }}\end{array}$ \\
\hline Fulvestrant & Faslodex & 2002 & 948 & 1248 \\
\hline $\begin{array}{l}\text { Paclitaxel albumin-stabilized } \\
\text { nanoparticle formulation }\end{array}$ & $\begin{array}{l}\text { Abraxane, nanoparticple } \\
\text { paclitaxel }\end{array}$ & 2005 & 5640 & 6837 \\
\hline Ixabepilone & Ixempra & 2007 & 6781 & 7742 \\
\hline Lapatinib ditosylate & Tykerb & 2007 & 3124 & 3567 \\
\hline Everolimus & Afinitor & 2009 & 7885 & 8701 \\
\hline Eribulin mesylate & Halaven & 2010 & 6193 & 6724 \\
\hline Ado-trastuzumab emtansine & Kadcyla & 2013 & 10,635 & 10,807 \\
\hline Pertuzumab & Perjeta & 2013 & 7758 & 7883 \\
\hline Palbociclib & Ibrance & 2015 & 10,690 & 10,677 \\
\hline
\end{tabular}

Bold values indicate additional value at lower or similar cost than existing treatments

Source: Memorial Sloan Kettering Cancer Center database: price and value of cancer drug

${ }^{\text {a }}$ Adjusted for inflation

Looking at an individual therapeutic class, two studies by Whalen et al. estimated the incremental cost per month of median OS (mOS) gained with the use of approved targeted therapies for colorectal cancer for first, second and third-line treatment of mCRC in Spain [16] and in France [17]. Both studies compared the treatment options bevacizumab, cetuximab, panitumumab, aflibercept and regorafenib and found the survival gain with targeted therapies to be higher in first-line than in second- or third-line treatment. The addition of a targeted agent gave the highest additional cost per month of OS gain in second-line treatment, followed by first-line treatment; the lowest cost per month of mOS gain was provided by third-line treatment. In fact, the latest entrant to the market—regorafenib-had the lowest cost per month of mOS gain in this analysis compared with cetuximab (with wild-type KRAS test) in third-line treatment (Fig. 1). The study showed that, from 2004 to 2015, the incremental cost per mOS gained did not appear to increase with US FDA approval date.

Although this analysis is simplistic (as it does not account for all the costs falling on the healthcare system), the observation that the OS benefit of regorafenib when coupled with its lower overall cost of treatment per month of OS gained represents an advance in value relative to established benchmarks and does challenge the perception that value for money is worsening progressively.

\section{The Role of Medicines in Driving Improvements in Cancer Survival}

Survival has improved significantly in some types of cancer. In both Europe and the USA, the 5-year survival rate has increased steadily over time across many cancer types, including breast, colorectal and ovarian cancer [18].
However, survival outcomes also vary significantly, and survival has remained stubbornly low in some cancers such as lung, pancreas and oesophageal cancers and most brain tumours [19].

Improvement in health outcomes can be attributed to a number of factors, including earlier diagnosis, allowing patients to be treated sooner; however, a recent study estimated that new medicines have accounted for a significant amount of the increase in cancer survival rates [10].

Advances from new classes and targeted therapies such as monoclonal antibodies have brought a fundamental change to our ability to treat cancer and have offered patients both extra years of life and a higher quality of life [20]. However, demonstrating the clinical value of a treatment is an ongoing process involving all aspects of how the treatment is used in different clinical environments and how it affects patients and the wider healthcare system.

\section{Are New Advances in Oncology Cost Effective?}

To assess value, we need to compare both the costs and the benefits. Given the modest survival benefits (in terms of days, weeks or months), despite the significant clinical advantages for patients in terms of additional quality of life, oncology products often struggle with cost-effectiveness assessment. It is argued that current methods of costeffectiveness analysis do not capture the full value of a product across all the dimensions that are important from the patient's perspective.

A study that looked at growth in the cost of medicines versus the value of health gains from 1998 to 2005 showed that although the cost of drug treatment for colorectal cancer rose, the new treatments also improved patient 


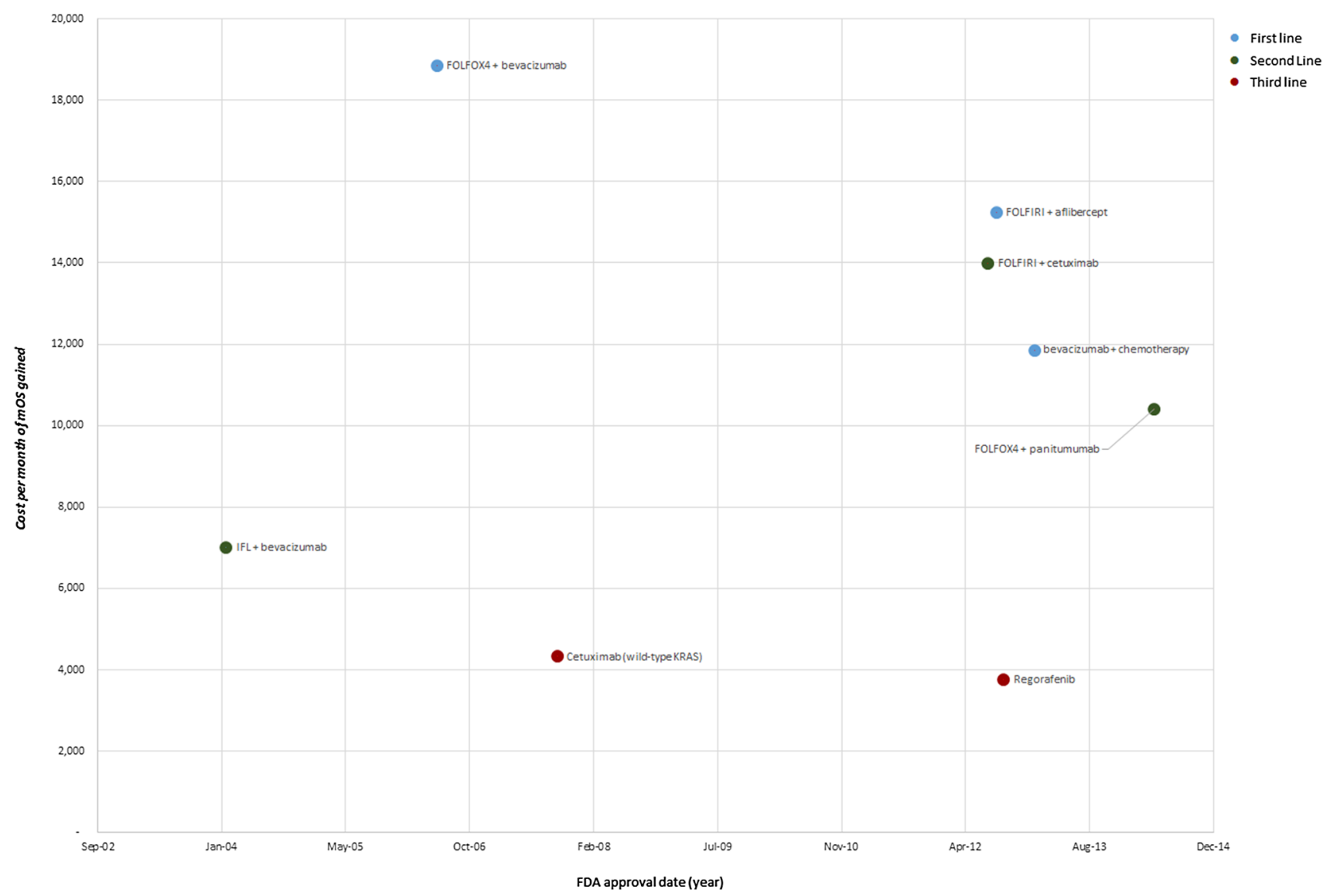

Fig. 1 Cost per month of median overall survival gained versus treatment approval date, in France $(€)$. Author's analysis based on data from Whalen et al. [17]. FOLFIRI folinic acid, fluorouracil and irinotecan, FOLFOX4 folinic acid, fluorouracil and oxaliplatin, IFL irinotecan, fluorouracil, and leucovorin, $m O S$ median overall survival

Fig. 2 Trends in cost of a 24-week colorectal cancer treatment regimen and change in quality-adjusted cost of care for colorectal cancer, 2000-2005. Author's analysis based on data by Lakdawalla et al. [21]

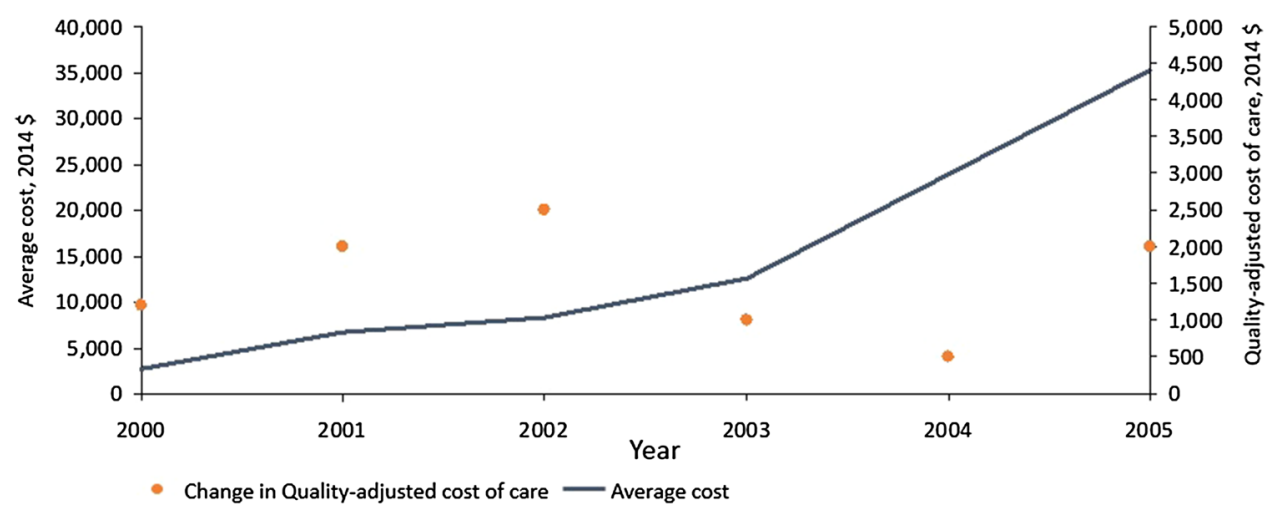

health outcomes [21]. In fact, the quality-adjusted cost of care remained largely flat because of roughly offsetting gains in health (Fig. 2). More specifically, whilst healthcare costs increased by $\$ U S 34,493$ as a result of the new medicines, health improved by 0.33115 quality-adjusted life-years (QALYs), valued at \$US33,115 per person. Thus, the quality-adjusted cost of care increased by only \$US1377 during this time period in the USA [21].

\section{Are New Advances in Oncology Unaffordable?}

The issue that concerns policy makers is not only the amount of money currently spent on healthcare but also the rate of increase in healthcare spending. Therapeutic oncology spending has increased in most developed nations, coinciding with the introduction of new biologics and targeted agents; however, the share of country-level 


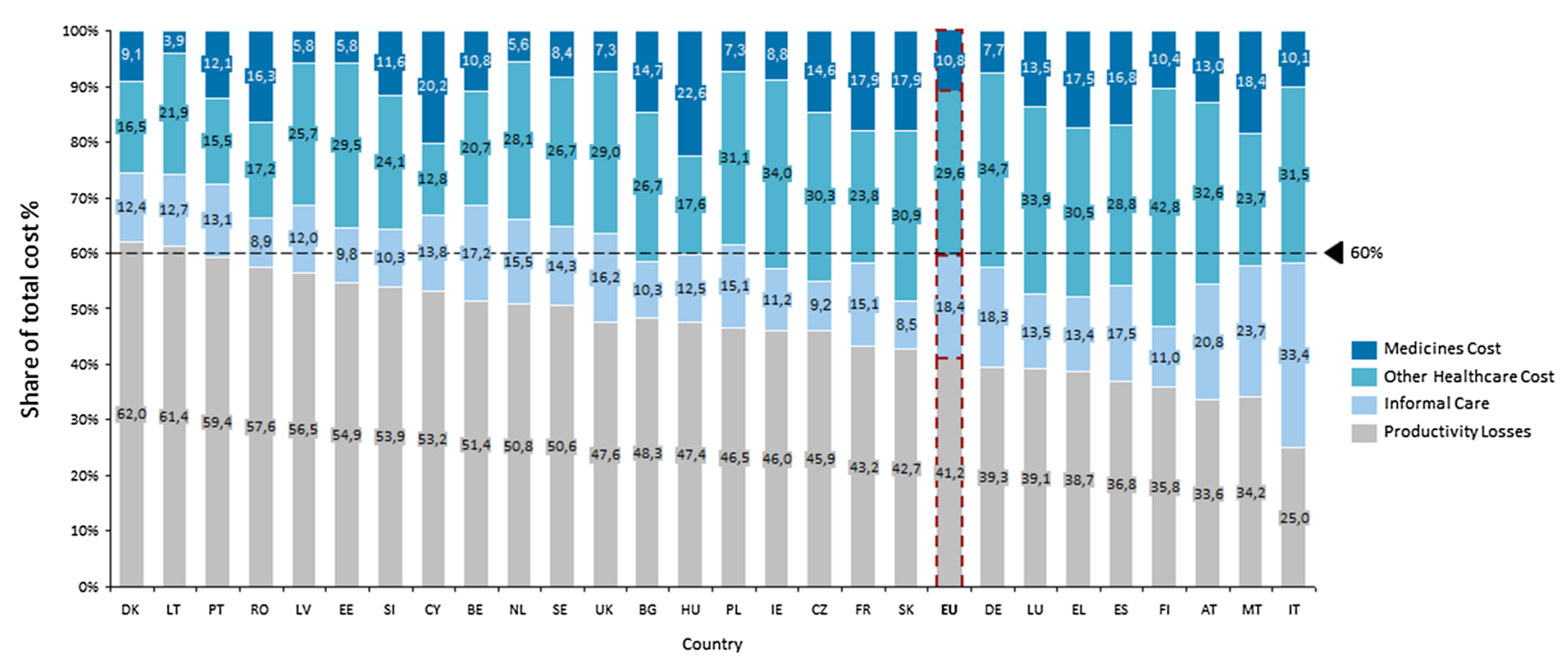

Fig. 3 Costs of cancer in the EU in 2009, by country (\%). Author's analysis based on data from Luengo-Fernandez et al. [23]

spending on cancer drugs relative to other drugs has only increased by about $1 \%$ [22].

While the development costs for cancer medicines account for some of the spending growth, not all increased costs are attributable to medicines; several other factors also play a significant role in driving increases in cost [22]. Indeed, if we consider the total costs associated with cancer, around $60 \%$ of total costs derive from productivity losses and informal care across the EU (Fig. 3), with inpatient costs accounting for the majority of healthcare costs. One study of cancer costs in Europe indicated that the main costs of cancer care accrued in the inpatient setting, representing, on average, $56 \%$ of cancer costs in the EU [23].

New cancer therapies are inevitably one of the main drivers of the rising cost of cancer care, but they need to be seen as only part of the cost burden associated with cancer, along with changes in population demographics such as aging, improvements in diagnosis and treatment rates, and increases in the average cost of other aspects of healthcare. There is also considerable variation in the share of medicines cost per country, which can be largely explained by differences in practice (prescribing behaviour, use of clinical/practice guidelines and readiness to adopt new/innovative medicines) as well as differences in reimbursement policies, pricing structures and the affordability ratio of new oncology medicines across the countries [24].

\section{Conclusions}

The aim of this paper was to test common assumptions associated with new oncology medicines. We acknowledge there is a general upward trend in the cost of pharmaceutical treatments in cancer, but we find tumour types for which the cost of new anticancer drugs is not increasing over time. This paper illustrates this with evidence from breast and colorectal cancer. We suggest that cancer drug prices depend on a number of factors, including pre-existing treatment options within a therapeutic class. Indeed, a number of studies have focused on the cost of treatment per month of OS gained as a simple (although partial) metric to judge value for money. Looking at cost per month of value gained, evidence for recent drugs for mCRC indicates that some new oncology drugs are delivering additional value at a lower cost than pre-existing products.

Given the importance of oncology products being used in combination, the development of similar approaches to capture the overall cost of treatment will be crucial. Alongside the development of more sophisticated approaches that can reflect the full value of new innovation, particularly at the end of a patient's life where treatments deliver incremental but vital benefit, metrics, as used in this paper, provide a useful comparison across products and between countries. These approaches need to be sufficiently tailored to deal with the different types of oncology products rather than applying a one-size-fits-all solution.

\section{Compliance with Ethical Standards}

Funding Financial support for the submitted work was provided by Bayer Pharmaceuticals Inc. Anthony Barron and Tim Wilsdon have received consulting fees from Bayer Pharmaceuticals Inc. for this research. Open access was funded by Bayer Pharmaceuticals Inc.

Conflict of interest Anthony Barron and Tim Wilsdon were commissioned to develop this analysis and they retain full editorial control on the resulting paper. Charles River Associates is an economic consultancy company with a long-established reputation for independent analysis. The views expressed herein are the views and opinions of the authors and do not reflect or represent the views of 
Charles River Associates or any of the organizations with which the authors are affiliated.

Open Access This article is distributed under the terms of the Creative Commons Attribution-NonCommercial 4.0 International License (http://creativecommons.org/licenses/by-nc/4.0/), which permits any noncommercial use, distribution, and reproduction in any medium, provided you give appropriate credit to the original author(s) and the source, provide a link to the Creative Commons license, and indicate if changes were made.

\section{References}

1. Danzon PM, Taylor E. Drug pricing and value in oncology. Oncologist. 2010;15(Suppl 1):24-31.

2. Experts in Chronic Myeloid Leukemia. The price of drugs for chronic myeloid leukemia (CML) is a reflection of the unsustainable prices of cancer drugs: from the perspective of a large group of CML experts. Blood. 2013;121(22):4439-42.

3. Ghinea N, Kerridge I, Lipworth W. If we don't talk about value, cancer drugs will become terminal for health systems. The Conversation. 2015 [online]. Available from: http://theconversation. com/if-we-dont-talk-about-value-cancer-drugs-will-becometerminal-for-health-systems-44072. Accessed 20 Oct 2016.

4. Kelly RJ, Smith TJ. Delivering maximum clinical benefit at an affordable price: engaging stakeholders in cancer care. Lancet Oncol. 2014;15(3):e112-8.

5. Cavalli F. An appeal to world leaders: stop cancer now. Lancet. 2013;381(9865):425-6.

6. Godman B, Malmström RE, Diogene E, et al. Are new models needed to optimize the utilization of new medicines to sustain healthcare systems? Expert Rev Clin Pharmacol. 2015;8(1):77-94.

7. Fojo T, Grady C. How much is life worth: cetuximab, non-small cell lung cancer, and the $\$ 440$ billion question. J Natl Cancer Inst. 2009;101(15):1044-8.

8. Kantarjian HM, Fojo T, Mathisen M, Zwelling LA. Cancer drugs in the United States: Justum Pretium-the just price. J Clin Oncol. 2013;31(28):3600-4.

9. Mailankody S, Prasad V. Five years of cancer drug approvals: innovation, efficacy, and costs. JAMA Oncol. 2015;1(4):539-40.

10. Howard DH, Bach PB, Berndt ER, Conti RM. Pricing in the market for anticancer drugs. J Econ Perspect. 2015;29(1):139-62.

11. Ramsey SD. How state and federal policies as well as advances in genome science contribute to the high cost of cancer drugs. Health Aff. 2015;34(4):571-5.
12. Barrett A, Roques T, Small M, Smith RD. How much will herceptin really cost? BMJ. 2006;333(7578):1118-20.

13. Haycox A. Why cancer? Pharmacoeconomics. 2016;34:625-7.

14. Santa-Maria CA, Gradishar WJ. Changing treatment paradigms in metastatic breast cancer: lessons learned. JAMA Oncol. 2015;1(4):528-34.

15. Pilon D, Queener M, Lefebvre P, Ellis LA. Cost per median overall survival month associated with abiraterone acetate and enzalutamide for treatment of patients with metastatic castrationresistant prostate cancer. J Med Econ. 2016;19(8):777-84.

16. Whalen J, Chang J, Ozer-Stillman I, Ambavane A, Ngai C. The cost of survival gain in metastatic colorectal cancer (mCRC) in Spain [abstract P-213]. Ann Oncol. 2015;26(Suppl. 4):iv62-iv62.

17. Whalen J, Chang J, Ozer-Stillman I, Ambavane A, Ngai C. The cost of survival gain in metastatic colorectal cancer (mCRC) in France [abstract P-212]. Ann Oncol. 2015;26(Suppl. 4):iv61-2.

18. De Angelis R, Sant M, Coleman MP, Francisci S. Cancer survival in Europe 1999-2007 by country and age: results of EUROCARE-5-a population-based study. Lancet Oncol. 2014;15(1):23-34. doi:10.1016/S1470-2045(13)70546-1.

19. Independent Cancer Taskforce. Achieving world-class cancer outcomes: a strategy for England 2015-2020, a report of the Independent Cancer Taskforce [online]. Available from: http:// www.cancerresearchuk.org/sites/default/files/achieving_worldclass_cancer_outcomes_-_a_strategy_for_england_2015-2020. pdf. Accessed 27 Sep 2016.

20. Weiner LM, Surana R, Wang S. Monoclonal antibodies: versatile platforms for cancer immunotherapy. Nat Rev Immunol. 2010;10(5):317-27.

21. Lakdawalla D, Shafrin J, Lucarelli C, Nicholson S, Khan ZM, Philipson TJ. Quality-adjusted cost of care: a meaningful way to measure growth in innovation cost versus the value of health gains. Health Aff. 2015;34(4):555-61.

22. IMS Institute for Healthcare Informatic. Developments in cancer treatments, market dynamics, patient access and value: global oncology trend report 2015. Available from: http://www.imshealth. com/en/thought-leadership/quintilesims-institute/reports/globaloncology-trend-2015\#ims-form.

23. Luengo-Fernandez R, Leal J, Gray A, Sullivan R. Economic burden of cancer across the European Union: a population-based cost analysis. Lancet Oncol. 2013;14(12):1165-74.

24. Ellen N, Newbould J, Conklin A. International variation in the usage of medicines: a review of the literature. Santa Monica, CA: RAND Corporation. 2010. Available from: http://www.rand.org/ pubs/technical_reports/TR830.html. 